\title{
Current situation on nutrient-drug interactions in health care practice
}

\author{
Conxita Mestres Miralles* \\ School of Health Sciences, Blanquerna-University Ramon Llull, Padilla 326, 08025 Barcelona, Spain
}

\begin{abstract}
Since the description of the serious interaction between IMAO drugs and tyramine containing foods in the 50s, there has been an incresing awarnes of their importanceof food-drug interactions as well as news ones are described and new drugs are continually apearing in the market. Furthemore, the impact of these interactions, increases due to the fact that polymedication is increasing, especially in older people.

We also have to take in account, in this area of knowledge the relation between nutritional status and drug treatment, as well as the adverse reactions that some drugs could have on it.

However we still facing some difficulties, such as:

- Lack of information in new commercialized drugs, due to the fact that these interactions are not studied or evaluated in premarketing assays

- Very few are described in patient's handout

- There are few databases and information sources, where health care professionals could find reliable and complete information.
\end{abstract}

Another important aspect is tha not always health care givers takes fully in account or give full importance to this kind of interaction. Therefore, there is still a long way to go, more reasearch in this area is needed in order to detect unknown food-drug. interactions and to have more knowledge about its importance in the patient outcome. Moreover ther is a need to increase the knowledge and awareness between physicians, pharmacists, dietitians and nurses, initiating them since the degree studies.

\section{Introduction}

A nutrient-drug interaction is defined as "as an alteration of kinetics or dynamics of a drug or a nutritional element, or a compromise in nutritional status as a result of the addition of a drug" [1] or as it states another definition "it is an interaction resulting from a physical, chemical, physiologic, or pathophysiologic relationship between a drug and a nutrient, multiple nutrients, food in general, or nutritional status" [2].

The awareness about interactions between drug and nutrition, began with the publication in 1941, about the influence of vitamin C deficiency on the effect of barbiturates [3], in the 60' an important food-drug interaction was described: IMA-tyramine containing foods that produced serious cases of hypertension and cerebrovascular accidents [4].

Since then other important interactions have been describes such those happening between divalent ions and tetracyclines [5] and more recently in early 90' the inhibition of metabolism of different drugs caused by grapefruit flavonones [6].

Over time there has been a more knowledge of the influence and interactions between pharmacologic treatment and nutrition. We can find different types of interactions deriving in a wide range of problems for the patient, due either for an increase or a decrease in the effect of the drug or deriving to a nutrient deficiency.

In Table 1, an approach of classification of different ways of interaction between nutrient and drugs and their possible consequences is given. Therefore, nutrient-drug or also said food-drug interactions include more mechanisms and consequences that the mere interference between food and drugs when taken simultaneously.

The increasing using of medicines, especially in elderly people and in chronic diseases, as well as the availability of new drugs, has also made this an area of special interest. Even though, that a long way has been done since 1941, I think that nowadays we still have several problems and issues to consider, in order to have a larger knowledge about nutrient-drug interactions, as well as how to manage them in the health care practice.

\begin{tabular}{|c|c|c|}
\hline Precipitating factor & $\begin{array}{c}\text { Object of the } \\
\text { interaction }\end{array}$ & Potential consequence \\
\hline Nutrition status & Drug & Treatment failure or drug toxicity \\
\hline Food or food component & Drug & Treatment failure or drug toxicity \\
\hline $\begin{array}{c}\text { Specific nutrient or other dietary } \\
\text { supplement ingredient }\end{array}$ & Drug & Treatment failure or drug toxicity \\
\hline Drug & Nutrition status & Altered nutrition status \\
\hline Drug & Specific nutrient & Altered nutrition status \\
\hline
\end{tabular}

Table 1. Classification of nutrient-drug interactions [2]

Correspondence to: Conxita Mestres Miralles, Dean, School of Health Sciences, Blanquerna-University Ramon Llull, Padilla 326, 08025 Barcelona, Spain, E-mail: concepciomm@blanquerna.url.edu

Received: May 27, 2017; Accepted: June 08, 2017; Published: June 12, 2017 


\section{Special areas of concern}

Nutrient-drugs interactions can potentially occur frequently, and if not considered or resolved can produce therapeutic failure or adverse reactions. But even though, nutrition-drugs interactions should always be takin in account, there are special situations where the consequences could be more serious.

- Elderly people. They are more exposed to interactions due that most of them are polymedicated, therefore we have more drugs that can potentially interact with nutrients. Also they situation of comorbidities and frailty make them more vulnerable to the consequences of the interactions.

- Treatments with narrow therapeutic index drugs. It is obvious that the effect of the interaction on one of these drugs leads to more serious consequences, due to inefficacity or toxicity.

Oral chemotherapy. In recent years there has been an increase in the marketing of antineoplastic drugs that can be administered orally. This has been a great improvement both in the increase of alternatives for cancer treatment as commodity for the patients that do not have to endure an intravenous administration. However, most of this new drugs have interactions with food, therefore, if they are not taken in account the effects of the drug can be compromised $[7,8]$.

Intensive care units (ICU). In this units the number of prescribed drugs to patients is usually high. Enteral nutrition is a preferred method of nutrition in this patients, that cannot take food orally, and administration of drugs through enteral feeding tubes, can lead to changes in the function of the gastrointestinal track thus producing nutritional problems, as well as problems of incompatibility between drugs and enteral nutrition. Due to the situation of patients in ICU, any deterioration of the nutritional status or problem with the drug effectivity or toxicity can be serious.

\section{Present problems and issues related to nutrient-drug interactions}

\section{Degree of knowledge about nutrient-drug interactions}

The actual proportion of adverse drug reactions due to nutrientdrug interactions is not known and, unfortunately, only when a serious adverse drug reaction follows the interaction does the matter receive a significant attention.

It is true that in the last decades there has been an increasing interest in the relation between nutrition and pharmacologic treatment, that has led to more research devoted to this area. Thus has implied that more publications (journal articles, books) addressing different problems occurring due to bidirectional effect between medicines and nutrition have appeared. However, the research in this area continues to be constricted to researchers that try to solve problems between food and drugs, arisen from the everyday work of health care professionals. Therefore, the existing information many times comes from anecdotal experience, have to be found in scattered journal publications, there is a scarce information about how to manage them and the information about their mechanism is limited and most of them are not collected and updated in databases easy to consult.

Widespread use of patient's medicines along with the broad variability in dietary habits, food composition and dietary supplements

The impact of nutrient-drug interactions is also increasing, due to different factors such as:
Increase in the use of medicines: The drug therapy is the most often used in any practice care. It has increased extensively with aging population, the prevalence of chronic diseases, emergence of new infectious diseases and expanding the number of effective drugs.

Dietary habits: Globalization has led to a change in dietary habits. Foods that some years ago where habitual only in certain areas or countries are now consumed worldwide. Therefore, when evaluating possible nutrient-drug interactions, we should have this into account and not limit us to consider common foods of our area.

Food composition and dietary supplements: There is a spreading use of functional drugs and nutraceuticals, that it makes more complicate the detection of possible nutrient-drug interactions. An interaction can be overlooked because we are not aware that in that food there is a component added, as for example calcium in fortified juices.

\section{Summary of product characteristics (SmPC), patient information leaflet (PIL)}

The summary of product characteristics (SmPC) of a medicine or the patient information leaflet (PIL), are important sources of information for healthcare professionals and patients. They should include the available information about interactions, however, they are not always optimally reported or described in them. In some cases they there is an incomplete agreement with the recommendations from the health authorities [9]. An study conducted in Spain, showed that the information about food-drug interactions provided in the SmPC was only present in $72.7 \%$ of the documents investigated, and that only in a $36 \%$ of the cases the information was described in the interaction section, although it is the correct place to appear in accordance with the recommendations form the health authorities [10].

\section{Knowledge level of the health care professionals}

In general healthcare professionals are not always aware of possible nutrient-drug interactions or their consequences. Some authors have explored the degree of knowledge of different healthcare professionals regarding food-drug interactions with similar results [11-13]. These findings showed that there was no relation between the degree of experience and the knowledge about food-drug interactions, and that more emphasis should be given to this area in health care training.

\section{How to improve}

As we have seen, there still many issues in nutrient-drug interaction to consider and be improved. Thus should include:

- Research: More research is needed in different directions. First a continuous one for determining possible nutrient-drug interactions, especially in drugs recently registered; and second to improve the knowledge of the clinical consequences of the interactions.

- To have bibliographic resources (data bases, etc.), that collect all the published results about nutrient-drug interactions, of easy access for healthcare professionals and patients.

- Improvement of the Summary of product characteristics and patient information leaflet

- To give more importance and incorporate nutrient-drug interactions issues during the process of new drugs development.

- Include more training in grade and postgrade of health sciences professionals 
- Increase interprofessional work, between physicians, pharmacists, nurses and dietitians

- Increase the patient and caregiver implication.

\section{References}

1. LB (2012) An introduction to: drug-nutrient interactions. In: IMER Meet March, Monash Univ.

2. Boullata JI, Hudson LM (2012) Drug-nutrient interactions: a broad view with implications for practice. J Acad Nutr Diet 112: 506-517. [Crossref]

3. Richards RK, Kueter K, Klatt TJ (1941) Effects of vitamin C deficiency on action of different types of barbiturates. Proc Soc Exp Biol Med 48: 403-409.

4. Asatoor AM, Levi AJ, Milne MD (1963) Tranylcypromine and cheese. Lancet 2: 733734. [Crossref]

5. Neuvonen PJ, Gothoni G, Hackman R, Björksten K (1970) Interference of iron with the absorption of tetracyclines in man. Br Med J 4: 532-534. [Crossref]

6. Bailey DG, Arnold JMO, Spence JD (1994) Grapefruit Juice and Drugs. Clin Pharmacokinet 26(2): 91-98.
7. Santana Martínez S, Marcos Rodríguez JA, Romero Carreño E (2015) Oral chemotherapy: food-drug interactions. Farm Hosp 39: 203-209. [Crossref]

8. Segal EM, Flood MR, Mancini RS, Whiteman RT, Friedt GA, et al. (2014) Oral chemotherapy food and drug interactions: A comprehensive review of the literature. $J$ Oncol Pract 10: e255-e268. [Crossref]

9. García (2001) Las interacciones con alimentos no se tratan bien en los prospectos. Correo Farm.

10. San Miguel MT, Martínez JA, Vargas E (2005) Food-drug interactios in the summary of products characteristics of proprietary medicinal products. Eur J Clin Pharmacol 61: 77-83. [Crossref]

11. Benni JM, Mk J, Tubaki BR, Renuka M (2012) Knowledge and awareness of food and drug interactions (FDI): a survey among health care professionals. Int J Pharmacol Clin Sci 1: 97-105.

12. Enwerem NM, Okunji P (2015) Knowledge, Attitudes and Awareness of Food and Drug Interactions among Nurses with Different Levels of Experience. Int $J$ Nurs 2: 1-9.

13. Couris RR, Tataronis GR, Dallal GE, Blumberg JB, Dwyer JT (2000) Assessment of healthcare professionals' knowledge about warfarin-vitamin $\mathrm{K}$ drug-nutrient interactions. J Am Coll Nutr 19: 439-445.

Copyright: $\odot 2017$ Miralles CM. This is an open-access article distributed under the terms of the Creative Commons Attribution License, which permits unrestricted use, distribution, and reproduction in any medium, provided the original author and source are credited. 